\title{
POD Model Order Reduction of Drift Diffusion Equations in Electrical Networks
}

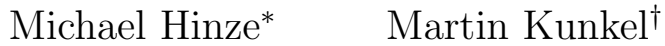 \\ Morten Vierling $\ddagger$ \\ Universität Hamburg \\ Department Mathematik \\ Bundesstr. 55, 20146 Hamburg, Germany
}

\begin{abstract}
In order to obtain a highly accurate model for integrated circuits it has been proposed in [1] to simulate the semiconductor components by a drift-diffusion equation. The coupling with the network equations then yields a nonlinear partial-differential algebraic equation (PDAE).

We discretize the drift-diffusion equations by standard finite elements as well as by Raviart-Thomas elements and simulate the resulting high dimensional DAE. We select snapshots of the semiconductor state variables from one or multiple simulations with different sets of parameters. From these snapshots a reduced state space approximation is obtained by using proper orthogonal decomposition (POD). We project the drift-diffusion equations onto the reduced state space yielding a nonlinear low-dimensional model for the drift-diffusion equations. This low-dimensional model then serves as surrogate for the driftdiffusion equations in the integrated circuit.

We present numerical examples which show that the POD-reduction of a semiconductor as a whole leads to an unstable ODE system for semiconductor device. This reduction strategy therefore seems not to be practicable. On the other hand, reducing the different state variables separately preserves the structure of the discretized equations and numerical simulations of the network with the reduced semiconductors are successful. We numerically analyze the behaviour of the reduction subject to parameter changes and different reduction levels.
\end{abstract}

\section{References}

[1] C. Tischendorf, Coupled Systems of Differential Algebraic and Partial Differential Equations in Circuit and Device Simulation, Habilitationsschrift, Mathematisch-Naturwissenschaftliche Fakultät, Humboldt-Universität Berlin, 2003.

\footnotetext{
*michael.hinze@math. uni-hamburg. de

$\dagger$ martin. kunkel@math. uni-hamburg. de

${ }^{\ddagger}$ morten. vierling@math. uni-hamburg. de
} 